of groups with considerable expertise in the field, in working with and trying to perfect the production, characterisation, and application of these reagents. I find the book full of useful information and to the further credit of the editors they not only maintain their ability to highlight the important areas of growth in the field, but also seem able to maintain a uniformity of style which, while providing the maximum of information, does so in a way which makes the text exciting and very readable. If the references seem out of date, this in no way detracts from the book since as a 'state of the art' text it does not need the 'hot off the press' label, but can rather be viewed as a solid, reliable treatise which is the 'gold standard' of its kind. I find nothing to criticise in it. This is an excellent book from which anyone vaguely interested in the field would gain immeasurably, and which anyone working in the field will find indispensable. I recommend it without reservation.

Alan M McGregor

\section{The Laboratory Diagnosis of Selected Inborn Errors of Metabolism}

By Vicky Allen Le Grys. (Pp 154; figures+tables. £28.50.) New York: Praeger Scientific. 1984.

This book contains a description of twelve inborn errors of metabolism. Contrary to what the title suggests, all aspects of the diseases including clinical features, biochemistry, pathophysiology, incidence, inheritance, and treatment, as well as laboratory diagnosis, are dealt with in some detail. Each section concludes with a typical case history. The text is readable, well presented, and adequately illustrated, but the standard of the information it contains is somewhat variable. There are a number of inaccurate and misleading statements and the current state of knowledge on a subject is not always to be found. This is almost inevitable when a single author attempts to write on diseases selected from such diverse fields.

There are some unusual features about the book's contents and their arrangement. It is rather surprising that some areas of the subject, like glycogen storage diseases and the organic acidurias, are not represented. On the other hand, it is difficult to justify the inclusion of neonatal hypothyroidism, as only a very small proportion of infants with this condition have an inborn error of metabolism. The contents are divided into two parts. The first of these describes those diseases for which screening tests are available, while the second part contains those for which screening is not routinely performed. The unsatisfactory nature of this classifica- tion is evident from the closing sentence on homocystinuria, which is in the former part of the book. It concludes that routine screening for this disease is not feasible. Conversely, screening for cystic fibrosis, glucose-6-phosphate dehydrogenase deficiency, or congenital adrenal hyperplasia is practised in many places, but they are all included in the latter part.

The various anomalies that are mentioned in the preceding paragraphs are probably a symptom of what seems to be a fundamental defect in the book, that the author appears not to have defined its objectives and purposes. It was left to the foreword writer to suggest a possible use by health scientists and physicians. However, such a restricted view of the subject is unsuitable for those who might be seriously involved with the diagnosis and management of patients with inborn errors of metabolism. It could provide a useful introduction for those taking the first parts of various medical and scientific postgraduate qualifications requiring a knowledge of inborn errors of metabolism, but even these readers would need to be aware of the narrow coverage by this text. It is an expensive book considering its limited use.

J B Holton

Immunogenetics. Its Application to Clinical Medicine Edited by $\mathrm{T}$ Sasazuki and $\mathrm{T}$ Tada. (Pp 308; figures + tables. $£ 21.00, \$ 30 \cdot 00$.) New York: Academic Press. 1984.

This book represents the proceedings of an international conference held in Kyoto, Japan in August 1983 on the implications for clinical medicine of recent advances in immunogenetics.

Inevitably the relationship between immunogenetics and disease has been explored most widely in animal models and this is reflected in the book by articles on murine models of systemic lupus erythematosus, myasthenia gravis, autoimmune thyroiditis, and allergic encephalitis. Nevertheless, headway is being made with human diseases as exemplified by HLA associations with immune response genes to streptococcal and cedar pollen antigens (Sasazuki $e t$ $a l$ ), with leprosy, and with the response to endorphin treatment in schizophrenia (van Rood et al).

In all there are 27 chapters divided into four sections: I. Immune response genes and disease. II. Cell interactions and activation in the human immune response. III. Genetic restrictions in the network and circuits. IV. Molecular genetics and biochemistry of immunologically important molecules. 